\title{
A study of coronary dominance and its clinical significance
}

\author{
D.P. Aricatt ${ }^{1}$, A. Prabhu², R. Avadhani ${ }^{1}$, K. Subramanyam³ ${ }^{3}$ A.S. Manzil' ${ }^{4}$, J. Ezhilan ${ }^{5}$, R. Das $^{6}$ \\ ${ }^{1}$ Department of Anatomy, Yenepoya Medical College, Yenepoya University Deralakatte, Mangalore, Karnataka, India \\ ${ }^{2}$ Yenepoya Research Centre, Yenepoya University Deralakatte, Mangalore, Karnataka, India \\ ${ }^{3}$ Department of Interventional Cardiology, K.S. Hegde Medical Academy Hospital, Mangalore, Karnataka, India \\ ${ }^{4}$ Department of CardioVascular Sciences, Sahakarana Hrudayalaya, Government Medical College Pariyaram, \\ Kannur, Kerala, India \\ ${ }^{5}$ Department of Cardiology, Madras Medical Mission, Chennai, Tamilnadu, India \\ ${ }^{6}$ Division of Data Analytics, Bioinformatics and Structural Biology (DABS), Mangalore, Karnataka, India
}

[Received: 23 October 2021; Accepted: 28 November 2021; Early publication date: 17 January 2022]

Background: Coronary artery disease is the most common cause of morbidity and mortality especially in the developing countries. The aim of the study was to find out cardiac dominance percentages and its association with coronary artery stenosis among each pattern of dominance. The objectives were to assess coronary vessel morphology of patients within each pattern of dominance, to find if gender differences exist among dominance patterns and also to find the distribution percentages of stenosis among dominance patterns.

Materials and methods: Four thousand angiograms from patients of Indian origin were studied prospectively after procuring the sanction for the same from the ethical committee of the pre-selected hospitals from four states of South India. Informed consents were obtained. Post coronary artery bypass grafting, post percutaneous coronary intervention patients and patient being diabetic for $\geq 5$ years were excluded from the study.

Results: Right cardiac dominance was seen in $85.5 \%$, left in 9.7\%, and co-dominant in $4.8 \%$ cases. The percentages of dominance were almost similar among both genders except for left dominance which were higher among male samples. The diameter of right coronary artery and left circumflex coronary artery coronary arteries were significantly associated with dominance patterns. The prevalence of stenosis was more for left dominance patterns, followed by right dominance patterns and least for co-dominant patterns.

Conclusions: There is a necessity to see association between dominance patterns with the coronary artery disease which can help the interventional cardiologists. The disease patterns in the present study were predominantly in the left dominant or in the co-dominant hearts. (Folia Morphol 2023; 82, 1: 102-107)

Key words: cardiac dominance, coronary vessel morphology, coronary artery disease 


\section{INTRODUCTION}

The major arteries supplying the heart are right coronary artery (RCA) and left main coronary arteries (LMCA) with the right posterior descending artery (PDA) for RCA and left anterior descending (LAD) and circumflex artery (LCX) for LMCA as the main branches [27]. Coronary dominance is described by which coronary artery branch gives off the PDA and supplies the inferior wall of heart. Estimates are that $80-85 \%$ of the population is right heart dominant with the posterior descending artery originating from the RCA; $7-13 \%$ of the population is left heart dominant with the PDA originating from the $L C x$, and about $2-5 \%$ is balanced or codominant with the PDA supplied by both the LCX and RCA [15]. When the branches from both the distal RCA and the distal LCX artery cross the inferior interventricular septum, it was termed as co-dominance. This was reported to have a prevalence of $7-8 \%$ [13]. Different studied by various authors confirm that the coronary artery system was right dominant in $85 \%$, left dominant in $8 \%$ and co-dominant in $7 \%$ of cases $[5,17,22,23]$. Certain recent studies indicated a high prevalence for left dominance followed by co-dominance pattern (DP) and lower prevalence percentages for right DP. This is contradictory to the previous studies $[1,11,25]$.

Ischaemic myocardial infarction and atherosclerotic involvement of LAD ostium were not associated with the left pattern of dominance [12]. The significance between dominance and the extent of coronary artery disease (CAD) were evaluated, by quantitative coronary angiography (QCA) it was found that CAD severity, arterial territory involvement and DP were related to each other [23]. In contrast, few authors reported the significant role of DP in the inferior wall infarcts and associated high mortality rates with left DP $[3,24]$.

Cardiac DPs with their correlations with atherosclerotic prominence and their clinical significance need to be studied in a broader perspective. Though left DP believed to have significantly higher mortality rate; supporting evidences are lacking due to reduced sample size. In this context, the present study outlooks the incidence of right, left and co-DP in a broader aspect. Present study reports the percentage prevalence of the DP and if gender specificity persists among each pattern of dominance. The present study tries to correlate the association between the diameters of the coronary artery and to the type of coronary vascular distribution.

\section{MATERIALS AND METHODS}

\section{Study population}

A cross sectional study was conducted in four cities of India. Hospitals were purposely selected according to the number of cardiac patients identified by them. The age of the study subjects was given a cut-off at 75 years due to marginal benefits marked during the follow-ups. Hence, a conservative approach is proven to be appropriate for the above-mentioned age which itself indicates a poor prognosis with an average yearly mortality rate of 33-35\% [4].The inclusion criteria were all patients who undergo percutaneous coronary angiographic procedure due to abnormalities in the normal cardiac parameters after obtaining their informed consent. Exclusion criteria were patients with a previous history of coronary artery bypass grafting and recanalised normal looking coronary arteries with or without in-stent restenosis coronary arteries as well as patients being diabetic for 5 or more than 5 years. The sample size was estimated by consulting a statistician and using the statistical software $G^{*}$ Power 3.0.10 and 4000 subjects were studied by convenience sampling. All ethical principles for human research were followed and ethical approval was obtained from the Institutional Ethics Committee of all the hospitals from where data was collected.

\section{Database pooling}

Four thousand angiogram reports were collected and studied. The three main DP are right dominant, left-dominant and balanced or co-dominant depending on the origin of the PDA from RCA or LCX. The origin of the PDA and the posterolateral branches were best evaluated in the left anterior oblique (LAO) cranial or anterior-posterior (AP) cranial view. If the $L C x$ is dominant, the optimal projection for the left PDA was the LAO cranial. Co-dominance can be explained as branches from both the distal RCA and the distal $L C x$ artery crosses the inferior interventricular septum. LAO cranial or AP cranial view can give an optimal projection to observe the same [15]. Normal or diseased segments among each DP were noted down.

Calibration assessments from QCA $[14,19]$ systems were carried out by the same method in which the coronary catheter was employed for angiography procedure. This was used as calibrating the object by automated edge detection technique resulting in corresponding calibration factors ( $\mathrm{mm} / \mathrm{pixel}$ ) and the vessel contour were detected by operator independent edge detection algorithms. Angiographic views were selected for calibration assessment by minimis- 


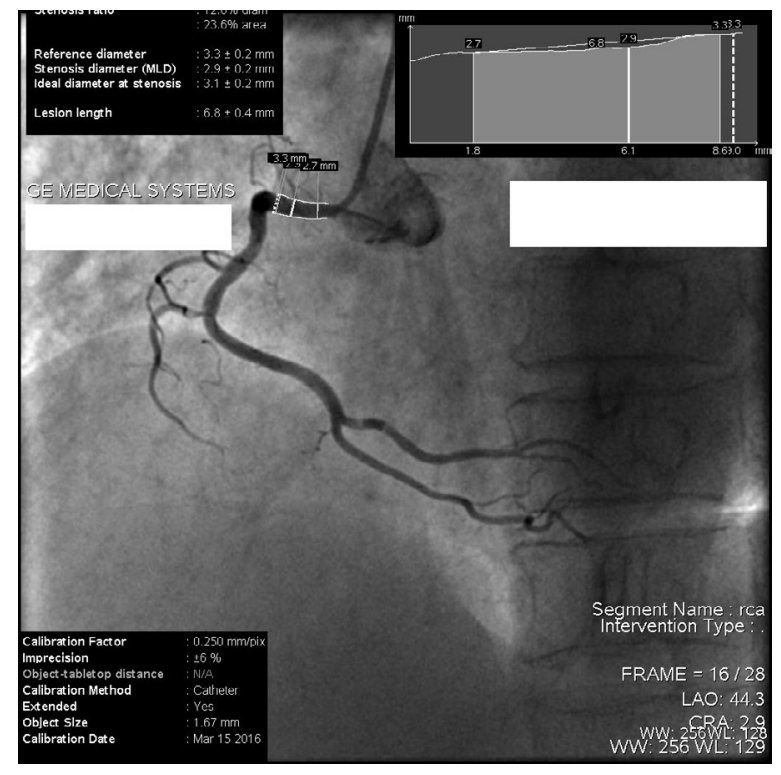

Figure 1. Quantitative coronary angiography image with an arrow pointing right coronary artery by left anterior oblique cranial projections. Image procured from DICOM image output on $100 \mathrm{M}$ bit Ethernet of GE Innova 2100 IO system with a capability of autosend and background transfer for fast transmission with minimal user interaction.

ing the foreshortening of the coronary segments by separating them from adjacent intervening structures.

For LCX ostial and proximal LCx were measured around ostium and before the origin of the first obtuse marginal for the proximal segment calibration assessment. For RCA, the ostium and proximal segments were measured before the origin of first acute marginal (AM1) by stenosis analysis programme using the automated coronary analysis package of the Innova 2100 IQ Cath at an AW4.4 workstation or Siemens QCA — Scientific coronary analysis. QCA were performed for vessel diameters ranging from $0.5 \mathrm{~mm}$ $-7 \mathrm{~mm}$ at syngo $X$ Workplace: VB2 1 with acquisition at 7.5, 10, 15 and $30 \mathrm{f} / \mathrm{s}$, acquisition for display and storage in original matrix of 12-bit (Figs. 1, 2). Statistical analysis of the present study was done using the SPSS software package for Windows version 22.0 (SPSS Inc., Chicago, IL). Descriptive statistics were used to present the socio-demographic data. Results were tabulated according to the standard format.

\section{RESULTS}

Among total sample population $(n=4000)$, there were 2,696 (67.4\%) males and 1,304 (32.6\%) females. Non flow limiting coronary arteries were seen in 933 (23.3\%) cases and 3,067 (76.7\%) had CAD.

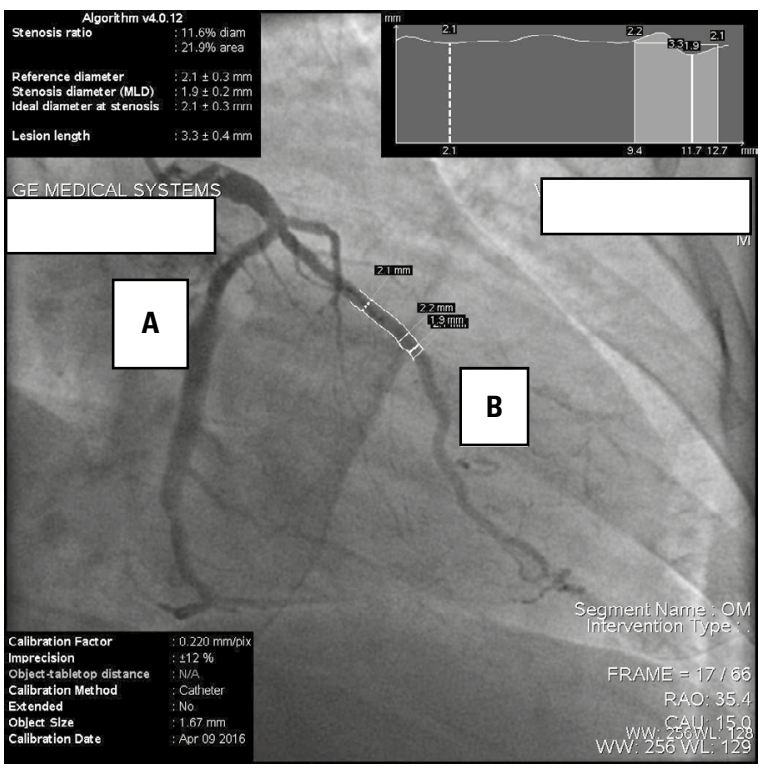

Figure 2. Quantitative coronary angiography images with arrows pointing left circumflex artery (A) and its obtuse marginal branch (B) by right anterior oblique caudal projections. Images procured from DICOM image output on $100 \mathrm{M}$ bit Ethernet of GE Innova 2100 IO system with a capability of autosend and background transfer for fast transmission with minimal user interaction.

\section{Coronary DP and significance}

There was no difference in the DP's of normal and diseased samples. The DP's were right dominant $>$ left dominant $>$ co-dominant. Among genderwise categorised samples DP's were right dominant $>$ left dominant $>$ co-dominant. The percentages of dominance were almost similar among both genders except for left dominance. Left dominance percentages were higher among male samples. These findings were similar to distribution of DP among total samples. The dimensions of coronary artery segments contributing for the DP namely, LCx and RCA were assessed. The coronary artery measurements (CAM) of normal segments were grouped under each DP for total and genderwise categorised samples. In total and genderwise categorised samples the difference in the CAM between LCx proximal part $(L C x-p)$ and RCA proximal part (RCA-p) in the right DP was highly significant $(p<0.01)$, and was very highly significant $(p<0.001)$ in left DP. However, this difference was not significant $(p>0.05)$ in co-DP (Table 1). Multiple comparisons were done for CAM of LCX-p and RCA-p with DP among total and genderwise categorised normal samples. Although, in total samples the difference in the CAM of LCx-p and RCA-p between right DP and left DP was very highly 
Table 1. Genderwise comparison of non-indexed coronary artery measurements (CAM) based on cardiac dominance patterns (DP) of normal samples $(n=933)$

\begin{tabular}{llccc}
\hline Samples & DP & LCx-p [mm] & RCA-p [mm] & P-value \\
\hline Male & Right dominant & $3.05 \pm 0.62$ & $3.19 \pm 0.66$ & $<0.01^{* *}$ \\
$(\mathrm{n}=521)$ & Left dominant & $3.39 \pm 0.58$ & $2.73 \pm 0.59$ & $<0.001^{* * *}$ \\
& Co-dominant & $3.19 \pm 0.58$ & $2.88 \pm 0.69$ & $>0.05$ \\
Female & Right dominant & $2.90 \pm 0.60$ & $3 \pm 0.61$ & $<0.01^{* *}$ \\
(n=412) & Left dominant & $3.30 \pm 0.56$ & $2.62 \pm 0.75$ & $<0.001^{* * *}$ \\
& Co-dominant & $3.04 \pm 0.70$ & $2.66 \pm 0.39$ & $>0.05$ \\
Total & Right dominant & $2.99 \pm 0.61$ & $3.10 \pm 0.64$ & $<0.01^{* *}$ \\
(n=933) & Left dominant & $3.35 \pm 0.57$ & $2.69 \pm 0.66$ & $<0.001^{* * *}$ \\
& Co-dominant & $3.13 \pm 0.63$ & $2.78 \pm 0.58$ & $>0.05$ \\
\hline
\end{tabular}

The CAM was taken based on quantitative coronary angiography reports and is represented as mean \pm standard deviation for each coronary artery segments in the DP's. Statistical test used: One way ANOVA; ${ }^{* * *} p<0.001$ indicates very highly significant difference; ${ }^{* *} p<0.01$ indicates highly significant difference; ${ }^{*} p<0.05$ indicates significant difference; $p>0.05$ indicates non-significant difference between non-indexed CAM of the segments contributing for the DP's. LCX-p — left circumflex coronary artery (proximal part); RCA-p — right coronary artery (proximal part)

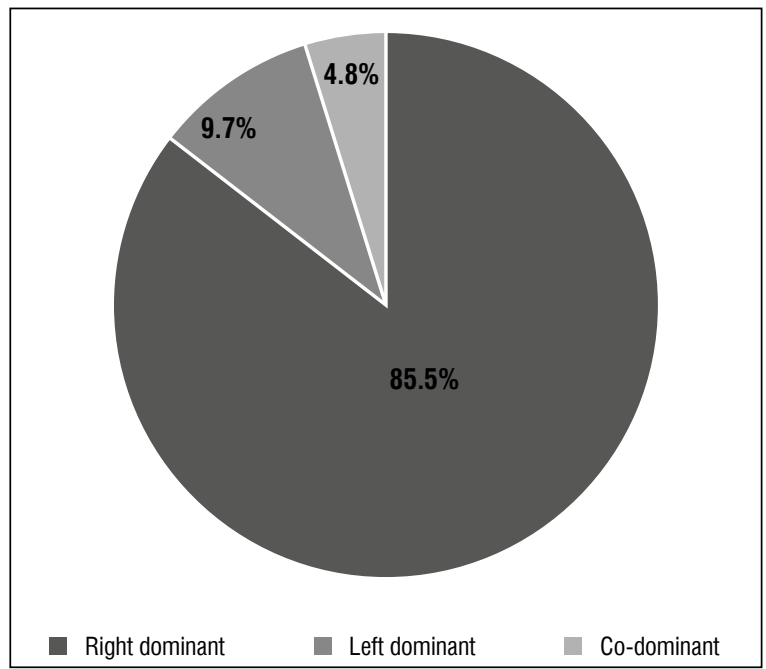

Figure 3. Distribution based on dominance patterns among total samples. The data was categorised based on quantitative coronary angiography reports.

Table 2. Genderwise multiple comparison of non-indexed coronary artery measurements (CAM) based on cardiac dominance patterns (DP) of normal samples $(\mathrm{n}=933)$

\begin{tabular}{|c|c|c|c|c|c|c|c|c|}
\hline \multirow{2}{*}{$\begin{array}{l}\text { Dependant } \\
\text { variable }\end{array}$} & \multicolumn{2}{|c|}{ DP } & \multicolumn{2}{|c|}{ Male samples } & \multicolumn{2}{|c|}{ Female samples } & \multicolumn{2}{|c|}{ Total samples } \\
\hline & & & MD & P-value & MD & P-value & MD & P-value \\
\hline \multirow[t]{3}{*}{ LCX-p } & $\mathrm{CO}$ & L & -0.20395 & 0.349 & -0.25885 & 0.291 & -0.22534 & 0.112 \\
\hline & $\mathrm{CO}$ & $\mathrm{R}$ & 0.13661 & 0.475 & 0.13986 & 0.569 & 0.14346 & 0.248 \\
\hline & L & $\mathrm{R}$ & $0.34057^{*}$ & $<0.01^{* *}$ & $0.39871^{*}$ & $<0.01^{* *}$ & $0.36880^{*}$ & $<0.001^{* * *}$ \\
\hline \multirow[t]{3}{*}{ RCA-p } & $\mathrm{CO}$ & L & 0.14324 & 0.643 & 0.03476 & 0.978 & 0.09608 & 0.698 \\
\hline & $\mathrm{CO}$ & $\mathrm{R}$ & $-0.31142^{*}$ & $<0.05^{*}$ & $-0.33945^{*}$ & $<0.05^{*}$ & $-0.32011^{*}$ & $<0.01^{* *}$ \\
\hline & $\mathrm{L}$ & $\mathrm{R}$ & -0.45466 & $<0.001^{* * *}$ & -0.37422 & $<0.01^{* *}$ & -0.41620 & $<0.001^{* * *}$ \\
\hline
\end{tabular}

The CAM was taken based on quantitative coronary angiography reports for each coronary artery segments in the DP's. Statistical test used: Post Hoc (Tukey's test), $\mathrm{p}<0.001^{* * *}$ indicates very highly significant difference, $p<0.01^{* *}$ indicates highly significant difference, $p<0.05^{*}$ indicates significant difference, $p>0.05$ indicates non-significant difference between non-indexed CAM of the segments contributing for the DP's. MD — mean difference; LCx-p — left circumflex coronary artery (proximal part); RCA-p — right coronary artery (proximal part); $\mathrm{L}$ - left dominant; $\mathrm{R}$ - right dominant; $\mathrm{CO}$ - co-dominant

significant ( $p<0.001)$, in genderwise categorised samples, it was only highly significant $(p<0.01)$. There was also a significant difference $(p<0.05)$ between co-dominant and right DP in genderwise categorised samples (Table 2 ).

\section{DISCUSSION}

\section{Cardiac dominance and associations with CAD}

In the present study there was right cardiac dominance in $85.5 \%$, left in $9.7 \%$, and co-dominant in $4.8 \%$ cases (Fig. 3, Table 3). Various authors have also reported the dominance percentages as $83-85 \%$ right, $6-8 \%$ left and $5-7 \%$ cases as co-dominant $[2,6,10,15,21,23,27,28]$. There was only slight variation in the co-dominant pattern which was lower in
Table 3. Distribution of normal, diseased and total segments based on dominance patterns (DP)

\begin{tabular}{lccc}
\hline DP & \multicolumn{3}{c}{ DP, $\mathbf{n}(\%)$} \\
\cline { 2 - 4 } & $\begin{array}{c}\text { Normal } \\
(\mathbf{n}=\mathbf{9 3 3})\end{array}$ & $\begin{array}{c}\text { Diseased } \\
(\mathbf{n}=\mathbf{3 0 6 7 )}\end{array}$ & $\begin{array}{c}\text { Total } \\
(\mathbf{n}=\mathbf{4 0 0 0})\end{array}$ \\
\hline Right dominant & $809(86.7 \%)$ & $2610(85 \%)$ & $3419(85.5 \%)$ \\
Left dominant & $74(7.9 \%)$ & $315(10.3 \%)$ & $389(9.7 \%)$ \\
Co-dominant & $50(5.4 \%)$ & $142(4.6 \%)$ & $192(4.8 \%)$ \\
\hline
\end{tabular}

The data was categorised based on quantitative coronary angiography reports and is represented as frequency with percentage in parenthesis; $n$ - frequency

the present study. However, the DP seen in other studies have not been consistent, especially in the ones that have looked at only left and right dominance and not the co-DP (Fig. 4). Gebhard et al. (2018) [10] 


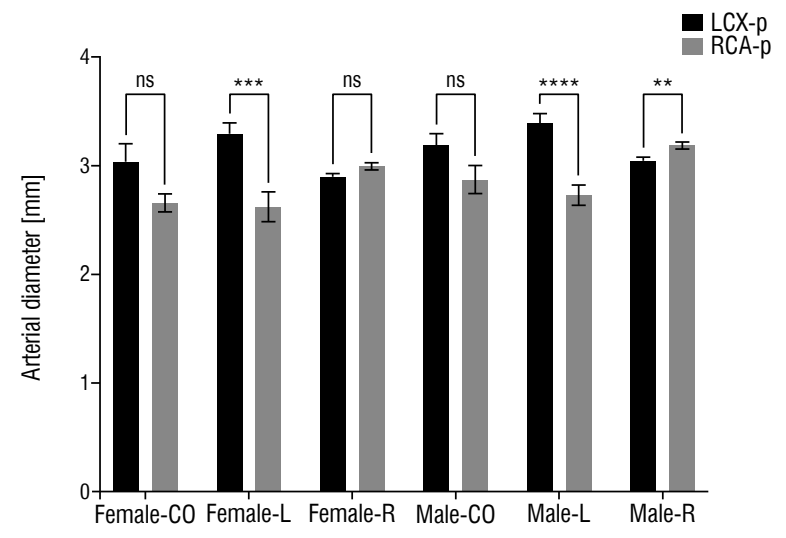

Figure 4. Artery measurements of dominance patterns among genderwise categorised samples. The data was categorised based on quantitative coronary angiography reports; LCx-p - left circumflex coronary artery (proximal part); RCA-p — right coronary artery (proximal part); $\mathrm{L}$ — left dominant; $\mathrm{R}$ — right dominant; $\mathrm{CO}-$ co-dominant; ${ }^{* * *} \mathrm{p}<0.001$ indicates very highly significant difference; ${ }^{* *} p<0.01$ indicates highly significant difference; ${ }^{*} p<0.05$ indicates significant difference; $p>0.05$ or ns indicates no significant difference.

denoted right dominance among $91 \%$ and left among $9 \%$ of the study population in their studies.

Another study found that left dominance was seen in $9.8 \%$ cases and non-left dominance in $90.2 \%$ samples. Few studies even found an opposite dominant pattern with a high prevalence for left dominance or for co-DP and lower prevalence percentages for right dominance $[10,20,22,25,26]$. There is a need to delve further into this aspect of coronary dominance as these variations should not merely be a consequence of different assessment methods used. Besides coronary angiography, the methods used have been 64 -slice computed tomography coronary angiography (CTCA), multidetector CTCA, corrosion casting or formalin-fixed human hearts.

In the present study the diameter of RCA-p and LCX-p coronary arteries were significantly associated with DP. The average size of the RCA-p was significantly smaller in the left DP as compared to that of right DP and vice versa for LCx-p dimensions among right dominant cases. Another Indian study conducted by Elangovan et al. (2005) [8] found similar association. Similarly the dimensions of $L C x$ were significantly smaller in co-dominant cases when compared to left DP $[7,16]$. Studies conducted in rest of the world have found similar results in relation to RCA-p and LCx-p sizes among the right and left DP. However, contrary to the present study they did not find association between CAM and co dominant patterns [16].
There is a necessity to see association between DP with the CAD as this can help the interventional cardiologist to be more alert. The disease patterns in the present study were predominantly in the left dominant or in the co dominant hearts. These findings are consistent with the studies that also found the similar disease patterns $[3,9,12]$. This left dominant disease pattern is biologically plausible because main origin of blood supply to the left ventricle is from the LCA. So it is regarded as the dominant vessel even in cases with anatomical right dominance.

Perfusion studies have also shown that even in anatomically right dominant hearts, left functional dominance is seen. Target lesions were more frequently found in hearts that had left coronary dominance with a higher incidence of periprocedural myocardial infarction [18]. Ghaffari et al. (2013) [11] have explained that atherosclerotic involvement of LAD was more related with atherosclerotic involvement of LAD ostium and ischemic myocardial infarction than to DP's. Contrary to this theory, few studies have shown more extensive CAD in patients with a right dominant coronary artery system [28] but it is not known whether they are exceptions or indicative of lack of association between DP and CAD. There has to be multi-centric studies with larger sample sizes to make a valid conclusion regarding the same.

\section{CONCLUSIONS}

The present study concludes that the prevalence of stenosis was more for left DP, followed by right DP and minimal for co-dominant patterns. A significant association was found between the diameters of the LCX-p and RCA-p and the type of coronary vascular distribution. There is a necessity to see association between DP with the CAD as this can help the interventional cardiologist to be more alert during the interventional procedures.

\section{Conflict of interest: None declared}

\section{REFERENCES}

1. Adil M, Nadeem M, Hafizullah M, et al. Comparison of left coronary artery diameter among diabetics and non-diabetics. J Postgrad Med Inst (Peshawar-Pakistan). 2012; 26(4): 369-376.

2. Altin C, Kanyilmaz $S$, Koc $S$, et al. Coronary anatomy, anatomic variations and anomalies: a retrospective coronary angiography study. Singapore Med J. 2015; 56(6): 339-345, doi: 10.11622/smedj.2014193, indexed in Pubmed: 25502334. 
3. Amin K, Javed M, Mehmood A, et al. Acute inferior wall myocardial infarction: Frequency of AV blocks. TPMJ. 2004; 11(1): 31-37.

4. Azad N, Lemay G. Management of chronic heart failure in the older population. J Geriatr Cardiol. 2014; 11(4): 329-337, doi: 10.11909/j.issn.1671-5411.2014.04.008, indexed in Pubmed: 25593582.

5. Bordoloi R. An anatomical study of coronary artery dominance in human cadaveric hearts. J Evid Based Med Healthcare. 2016; 3(103): 5695-5699, doi: 10.18410/ jebmh/2016/1177.

6. Cademartiri F, Malagò R, La Grutta L, et al. Coronary variants and anomalies: methodology of visualisation with 64-slice CT and prevalence in 202 consecutive patients. Radiol Med. 2007; 112(8): 1117-1131, doi: 10.1007/ s11547-007-0210-0, indexed in Pubmed: 18080097.

7. Dhakal A, Shrestha R, Maskey A, et al. Coronary artery dimensions in the Nepalese population. JASI. 2015; 64(1): 27-31, doi: 10.1016/j.jasi.2015.03.001.

8. Elangovan $C$, Jaganathan V, Alageshan $R$, et al. Clinical and anthropometric correlation of normal Coronary artery dimensions. Indian Heart J. 2005; 57(5): 381-425.

9. Gebhard C, Fuchs TA, Stehli J, et al. Coronary dominance and prognosis in patients undergoing coronary computed tomographic angiography: results from the CONFIRM (COronary CT Angiography EvaluatioN For Clinical Outcomes: An InteRnational Multicenter) registry. Eur Heart J Cardiovasc Imaging. 2015; 16(8): 853-862, doi: 10.1093/ ehjci/jeu314, indexed in Pubmed: 25744341.

10. Gebhard C, Gick M, Ferenc M, et al. Coronary dominance and prognosis in patients with chronic total occlusion treated with percutaneous coronary intervention. Catheter Cardiovasc Interv. 2018; 91(4): 669-678, doi: 10.1002/ ccd.27174, indexed in Pubmed: 28795482.

11. Ghaffari S, Kazemi B, Dadashzadeh J, et al. The relation between left coronary dominancy and atheroscleroticinvolvement of left anterior descending artery origin. J Cardiovasc Thorac Res. 2013; 5(1): 1-4, doi: 10.5681/ jcvtr.2013.001, indexed in Pubmed: 24251001.

12. Goldberg A, Southern DA, Galbraith PD, et al. Coronary dominance and prognosis of patients with acute coronary syndrome. Am Heart J. 2007; 154(6): 1116-1122, doi: 10.1016/j.ahj.2007.07.041, indexed in Pubmed: 18035084.

13. Helft G, Dambrin G, Zaman A, et al. Percutaneous coronary intervention in anticoagulated patients via radial artery access. Catheter Cardiovasc Interv. 2009; 73(1): 44-47, doi: 10.1002/ccd.21758, indexed in Pubmed: 19089936.

14. Hermiller JB, Cusma JT, Spero LA, et al. Quantitative and qualitative coronary angiographic analysis: review of methods, utility, and limitations. Cathet Cardiovasc Diagn. 1992; 25(2): 110-131, doi: 10.1002/ccd.1810250207, indexed in Pubmed: 1544153.

15. Jeffrey JP, Scott K, and De. Coronary arteriography and Intra coronary Imaging. In: Mann DL, Zipes DP, Libby
P, Bonow RO. Braunwald's heart disease: a textbook of cardiovascular medicine. Part 1. Elsevier Health Sciences 2017: 392-428.

16. Kim E, Yoo J, Cheon W, et al. Coronary artery size in Korean: normal value and its determinants. Korean Circ J. 2005; 35(2): 115, doi: 10.4070/kcj.2005.35.2.115.

17. Koşar $P$, Ergun E, Oztürk $C$, et al. Anatomic variations and anomalies of the coronary arteries: 64-slice CT angiographic appearance. Diagn Interv Radiol. 2009; 15(4): 275-283, doi: 10.4261/1305-3825.DIR.2550-09.1, indexed in Pubmed: 19957241.

18. Lam MK, Tandjung K, Sen H, et al. Coronary artery dominance and the risk of adverse clinical events following percutaneous coronary intervention: insights from the prospective, randomised TWENTE trial. Eurolntervention. 2015; 11(2): 180-187, doi: 10.4244/EIJV11I2A32, indexed in Pubmed: 24602919.

19. MacAlpin RN, Abbasi AS, Grollman JH, et al. Human coronary artery size during life. A cinearteriographic study. Radiology. 1973; 108(3): 567-576, doi: 10.1148/108.3.567, indexed in Pubmed: 4269246.

20. Moore KL, Dalley AF, Agur AM. Clinically oriented anatomy. Lippincott Williams \& Wilkins 2013: 151-174.

21. Mowatt G, Cummins E, Waugh N, et al. Systematic review of the clinical effectiveness and cost-effectiveness of 64-slice or higher computed tomography angiography as an alternative to invasive coronary angiography in the investigation of coronary artery disease. Health Technol Assess. 2008; 12(17): iii-iv, ix, doi: 10.3310/hta12170, indexed in Pubmed: 18462576.

22. Paudel N, Jha GS, Alurkar VM, et al. Coronary dominance and predictors of adverse events during coronary interventional procedures: an observational study. J Adv Intern Med. 2017; 6(1): 4-8, doi: 10.3126/jaim.v6i1.18311.

23. Paul A, Avadhani R, Subramanyam K. Anomalous origins and branching patterns in coronary arteries: an angiographic prevalence study. JASI. 2016; 65(2): 136-142, doi: 10.1016/j.jasi.2016.09.001.

24. Reagan K, Boxt LM, Katz J. Introduction to coronary arteriography. Radiol Clin North Am. 1994; 32(3): 419-433, indexed in Pubmed: 8184022.

25. Reddy V, Lokanadham S. Coronary dominance in south Indian population. Int J Med Res Health Sci. 2013; 2(1): 78-82.

26. Shukri IG, Hawas JM, Karim SH, et al. Angiographic study of the normal coronary artery in patients attending Ulaimani Center for Heart Diseases. ESJ. 2014; 10(24): 384-415.

27. Standring S, Borley NR, Collins P, Crossman AR, Gatzoulis MA, Healy JC. Gray's Anatomy. 41 stedn. Churchill Livingstone, London 2008: 978-980.

28. Vasheghani-Farahani A, Kassaian SE, Yaminisharif $A$, et al. The association between coronary arterial dominancy and extent of coronary artery disease in angiography and paraclinical studies. Clin Anat. 2008; 21(6): 519-523, doi: 10.1002/ca.20669, indexed in Pubmed: 18698656. 